\title{
Predictive value of immuno-inflammatory and nutritional measures modulated by neoadjuvant chemotherapy on the response of neoadjuvant chemotherapy and long-term outcomes in patients with esophageal cancer
}

\author{
YUSUKE ISHIBASHI, HIRONORI TSUJIMOTO, SHUICHI HIRAKI, KEITA KOUZU, \\ SATOSHI TSUCHIYA, YUJIRO ITAZAKI, YOSHIHISA YAGUCHI, HIROYUKI HORIGUCHI, \\ SHINSUKE NOMURA, NOZOMI ITO, EIJI SHINTO, YOJI KISHI and HIDEKI UENO \\ Department of Surgery, National Defense Medical College, Tokorozawa, Saitama 359-8513, Japan
}

Received July 25, 2019; Accepted November 8, 2019

DOI: $10.3892 / \mathrm{ol} .2019 .11122$

\begin{abstract}
It has been reported that immuno-inflammatory and nutritional parameters are associated with long-term survival in various malignancies. However, little is known regarding the associations between alterations of these parameters during neoadjuvant chemotherapy (NAC) and the response to NAC in patients with esophageal cancer. The present study examined the clinical significance of alterations in these parameters during NAC in terms of the response to NAC and the long-term outcomes in patients with esophageal cancer. Various systemic immuno-inflammatory and nutritional measures including the systemic neutrophil-to-lymphocyte ratio (NLR), platelet-to-lymphocyte ratio (PLR), C-reactive protein (CRP)-to-albumin ratio (CAR) and psoas muscle index (PMI) were examined before and after NAC. Statistical analyses were performed to determine the significance of immuno-inflammatory and nutritional parameters prior to NAC and alterations during NAC regarding the response to NAC and long-term outcomes. The NLR, PMI, neutrophil count and platelet count declined significantly following NAC, whereas no alterations in PLR, CAR, lymphocyte counts, CRP levels and albumin concentration were observed. The decreases in NLR and neutrophil counts following NAC were strongly associated with a favorable overall survival $(\mathrm{P}=0.006)$. In conclusion, decreases in NLR and neutrophil counts following NAC were clinically significant predictors of the response to NAC and of survival in esophageal cancer, respectively.
\end{abstract}

Correspondence to: Professor Hironori Tsujimoto, Department of Surgery, National Defense Medical College, 3-2 Namiki, Tokorozawa, Saitama 359-8513, Japan

E-mail: tsujihi@ndmc.ac.jp

Key words: neutrophil-to-lymphocyte ratio, platelet-to-lymphocyte ratio, C-reactive protein-to-albumin ratio, psoas muscle index, esophageal cancer, neoadjuvant chemotherapy

\section{Introduction}

Esophageal cancer is the eighth type of cancer most frequently diagnosed and the sixth leading cause of death in the world (1). Despite advances in surgical techniques, postoperative managements, and treatment strategies, the mortality rate of esophageal cancer remains high and the 5-year survival rate was reported to range from 15 to $25 \%(2,3)$. In Japan, neoadjuvant chemotherapy (NAC) with a combination of cisplatin plus 5-fluorouracil (FP) followed by surgery is a standard treatment strategy for resectable stage II/III esophageal squamous cell carcinoma (ESCC) (4). However, in patients who respond unfavorably, the inefficient preoperative therapy should be discontinued and surgery should not be delayed. Conversely, patients who respond favorably to preoperative therapy may benefit from additional preoperative treatment. Thus, it is important to establish a method that may be used to predict the pathological response to NAC reliably to prevent wasting time and improve surgical outcomes.

Recently, several systemic immuno-inflammatory responses have been reported to be independent prognostic biomarkers in many types of malignancies, such as stomach, colon, hepatic, lung, and esophageal cancers (5-11). However, the clinical impact of these systemic immuno-inflammatory responses modulated by NAC on the response to NAC for esophageal cancer remains unclear.

In this study, we investigated the clinical significance of changes in systemic immuno-inflammatory measures during $\mathrm{NAC}$ in terms of the response to NAC and long-term outcomes in patients with esophageal cancer.

\section{Materials and methods}

Patients. A total of 85 consecutive patients underwent a transthoracic esophagectomy for clinical stage II or III esophageal cancer at the National Defense Medical College Hospital (Saitama, Japan) between January 2009 and December 2014. All patients were administered NAC before esophagectomy. Among the 85 patients, 9 (11\%) were women and 76 (89\%) were 
men. The mean age was $68.6 \pm 0.9$ years (range, $43-86$ years). The tumor node metastasis criteria from the eighth edition of the Union for International Cancer Control classification system were used for tumor staging (12). All patients were intended to be administered 2 courses of NAC before esophagectomy. Patients who failed to complete 2 courses of NAC were excluded from this study. All patients including clinically stage IV were considered to be resectable after NAC and underwent the resection with curative intent. Patients who underwent the palliative surgery, such as salvage surgery or bypass surgery, were excluded from this study. Among the 85 patients, 7 patients could not achieve oral intake because of the obstructing locally advanced esophageal cancer, and underwent laparoscopic jejunostomy and adequate enteral nutrition during NAC (13).

Neutrophil count, platelet count, lymphocyte count, CRP level, albumin concentration, and psoas mass index (PMI) were evaluated before and after NAC. The neutrophil-to-lymphocyte ratio (NLR) was defined as the absolute neutrophil count divided by the absolute lymphocyte count, the platelet-to-lymphocyte ratio (PLR) was defined as the absolute platelet count divided by the absolute lymphocyte count, and the C-reactive protein-to-albumin ratio (CAR) was calculated by dividing the serum CRP level by the serum albumin level. PMI $\left(\mathrm{cm}^{2} / \mathrm{m}^{2}\right)$ was calculated by dividing the area of the psoas muscle at the third lumbar vertebra in a cross-sectional computed tomography (CT) image by height squared as previously described (14). The blood test after NAC was performed one month after the last chemotherapy and just before the operation. No patients were administered granulocyte-colony stimulating factor during NAC, which should affect the immuno-inflammatory markers.

The association between patient demographics (information obtained from our computer database) and overall survival (OS) was evaluated in patients with esophageal cancer. OS was measured from the date of esophagectomy to the date of death from any cause. Patients who survived were defined to be censored in our survival analyses. All patients were observed at our hospital or outpatient clinic at 3- to 4-month intervals during the first two years of the study, and every 6 or 12 months thereafter for three years. A CT scan was performed, and tumor markers were assessed every 6 months until five years after the resection. Five years after surgery, annual follow-ups were conducted by telephone with the patients, the patient's family members, or their practitioners. The Institutional Review Board of the National Defense Medical College Hospital approved this protocol. All patients provided written informed consent prior to their inclusion in the study.

NAC and surgical procedure. All patients were intended to be administered two courses of NAC before esophagectomy. FP chemotherapy was repeated twice every 3 weeks with a dose of $80 \mathrm{mg} / \mathrm{m}^{2}$ of cisplatin was given by intravenous drip infusion on day one and 5-fluorouracil was administered at a dose of $800 \mathrm{mg} / \mathrm{m}^{2}$ by continuous infusion on days one through five. Esophageal resection was performed approximately one month after the last infusion.

In the open surgery patients, open transthoracic esophagectomy was performed through the right fifth or sixth thoracotomy with adequate lymphadenectomy. In the video-assisted surgery patients, thoracoscopic esophagectomy was performed using three ports with diameters of $12 \mathrm{~mm}$ as described previously (15). Gastric tube reconstruction was performed by laparoscopy-assisted surgery in all patients (16).

Histopathological response assessment. Histopathological response was graded into five groups. Grade 0 response indicated no evidence of effect, Grade la response indicated very slight effect (viable tumor cells occupy more than $2 / 3$ of the tumorous area), Grade $1 \mathrm{~b}$ response indicated slight effect (viable tumor cells remain in more than $1 / 3$ but less than $2 / 3$ of the tumorous area), Grade 2 response indicated considerable effect (viable tumor cells remain in less than $1 / 3$ of the tumorous area), and Grade 3 response indicated complete response (12).

Statistical analysis. Statistical analyses were performed using the Mann-Whitney $U$ or chi-square tests. The Kaplan-Meier method was used to make the OS curve, and the survival differences were compared with the log-rank test. A multivariate analysis was performed with the Cox proportional hazards model, and prognostic variables were introduced in the model when the univariate analysis revealed a significance level of $\mathrm{P}<0.05$. All values with $\mathrm{P}<0.05$ were statistically significant. $\mathrm{A}$ time-dependent receiver operating characteristics (ROC) curve for censored survival outcomes was constructed to estimate the optimal cutoff value of the inflammatory-nutritional measures before NAC in Tables I and II. JMP 12.0 (SAS Institute Inc., Cary, NC, USA) was used to perform all analyses.

\section{Results}

The prediction of the pathological responses using pretherapeutic inflammatory-nutritional measures is presented in Table I. The pretherapeutic NLR was a significant predictive marker of the pathological response to NAC. However, pretherapeutic PLR, CAR, and PMI had no influence on pathological responses to NAC. Among the individual components of the systemic immuno-inflammatory parameters, high neutrophil counts before NAC were associated with a favorable pathological response to NAC (Table II). However, this correlation was not observed with lymphocyte, platelet, CRP, and albumin levels.

The NLR and PMI decreased significantly after NAC; such decreases were not observed in the PLR and CAR (Fig. 1). Additionally, the neutrophil and platelet counts were significantly decreased after NAC. However, no changes in lymphocyte count, CRP levels, and albumin levels were reported during NAC (Fig. 2).

Clinicopathological characteristics were compared between patients grouped based on changes in the NLR, PLR, CAR, and PMI after NAC (Table III). Patients with a decreased NLR after NAC had pStage III/IV diseases more frequently. Additionally, patients with a decreased CAR after NAC had a better histopathological response to NAC compared to those with an increased CAR. There were no correlations among gender, age, surgical procedure, tumor depth, lymph node metastasis, tumor location, histological type, tumor differentiation, or response to NAC with the 

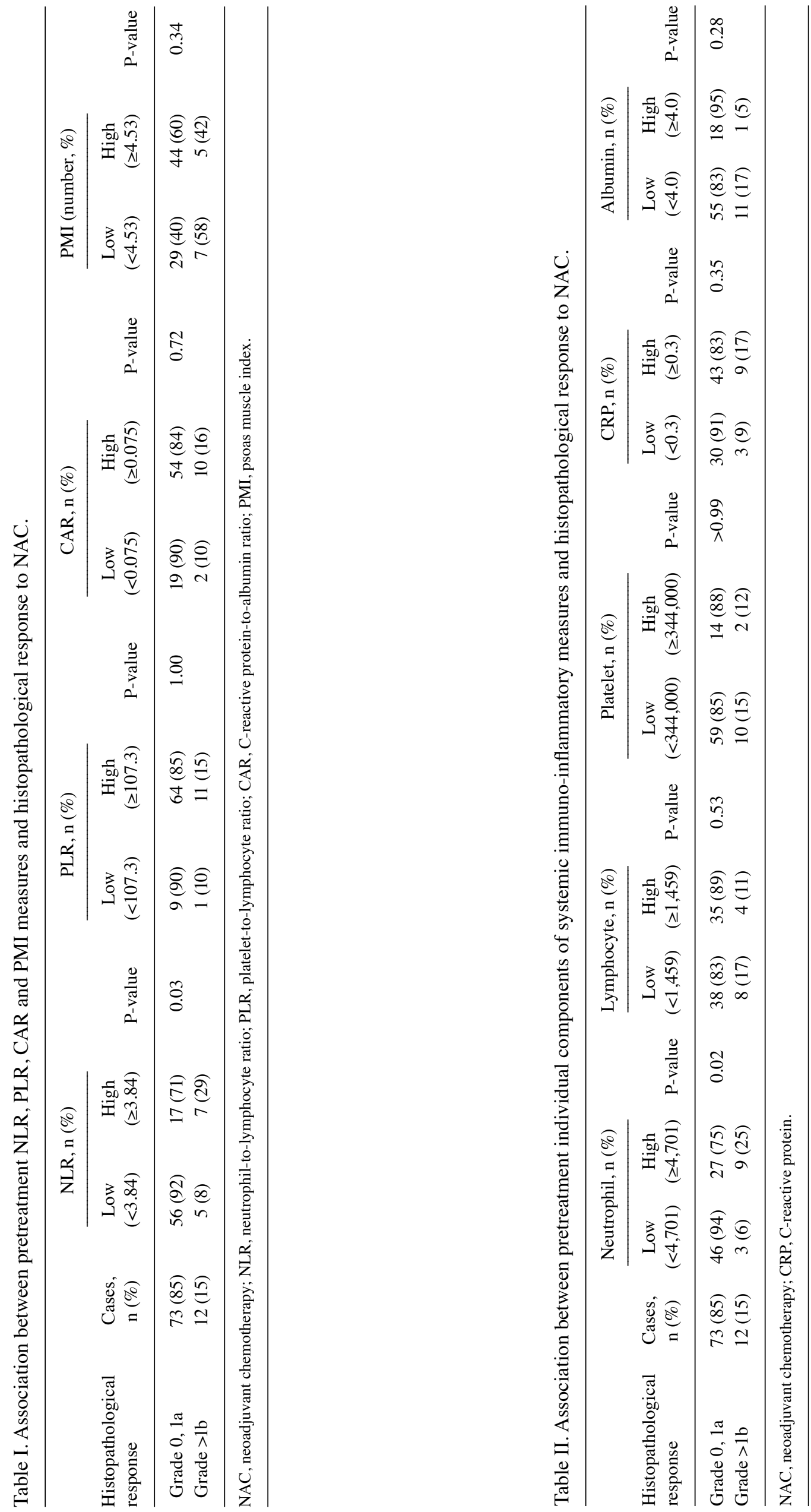

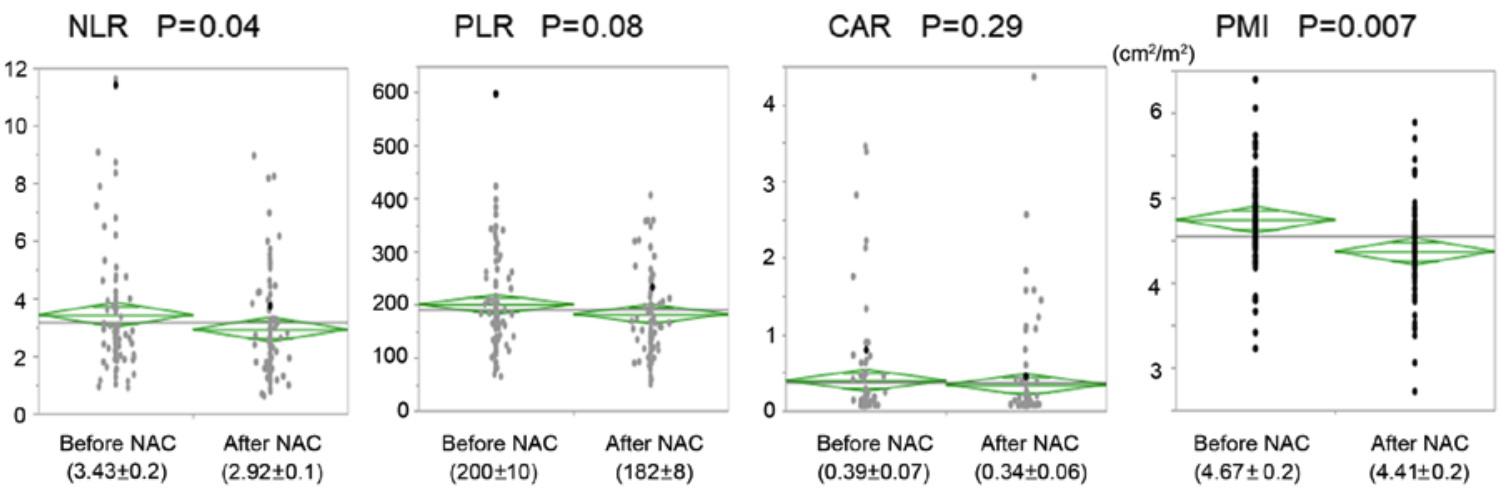

Figure 1. Numerical data on NLR, PLR, CAR and PMI before and after NAC. NLR, neutrophil-to-lymphocyte ratio; PLR, platelet-to-lymphocyte ratio; CAR, C-reactive protein-to-albumin ratio; PMI, psoas muscle index; NAC, neoadjuvant chemotherapy.
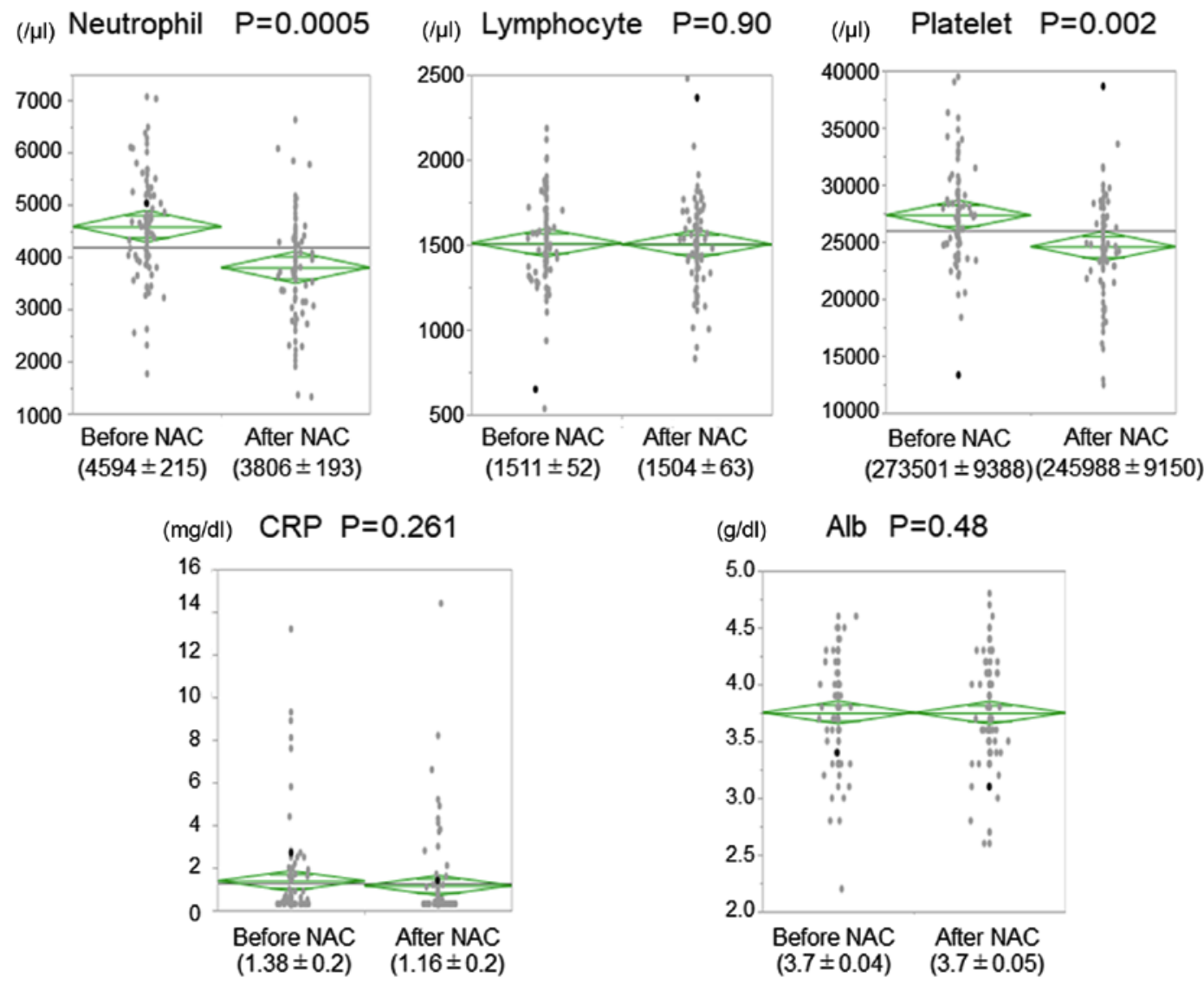

Figure 2. Numerical data on neutrophils, platelets, lymphocytes, CRP levels and albumin levels before and after NAC. CRP, C-reactive protein; NAC, neoadjuvant chemotherapy.

changes in the NLR, PLR, CAR, and PMI after NAC. There were no correlations between the postoperative complications and the changes in the NLR, PLR, CAR, and PMI after NAC (Table SI).

Clinicopathological characteristics were compared between patients who were grouped according to changes in the individual components of the systemic immuno-inflammatory measures, i.e., neutrophil, lymphocyte, and platelet counts; and CRP and albumin levels after NAC (Table IV). There were no relationships between clinicopathological features and changes in the individual components of the systemic immuno-inflammatory measures, except that patients with decreased neutrophil counts after NAC had a better histological response.

Patients with a decreased NLR after NAC had a significantly favorable OS compared to those with an increased NLR; however, such differences were not observed with changes in the PLR, CAR, and PMI (Fig. 3). Similarly, patients with a decreased neutrophil count after NAC had a significantly favorable OS compared to those with an increased neutrophil count; however, such differences were not observed in the changes in lymphocyte and platelet counts and CRP and albumin levels (Fig. 4).

Univariate and multivariate analyses of factors that might affect OS were performed separately for immune-inflammatory 


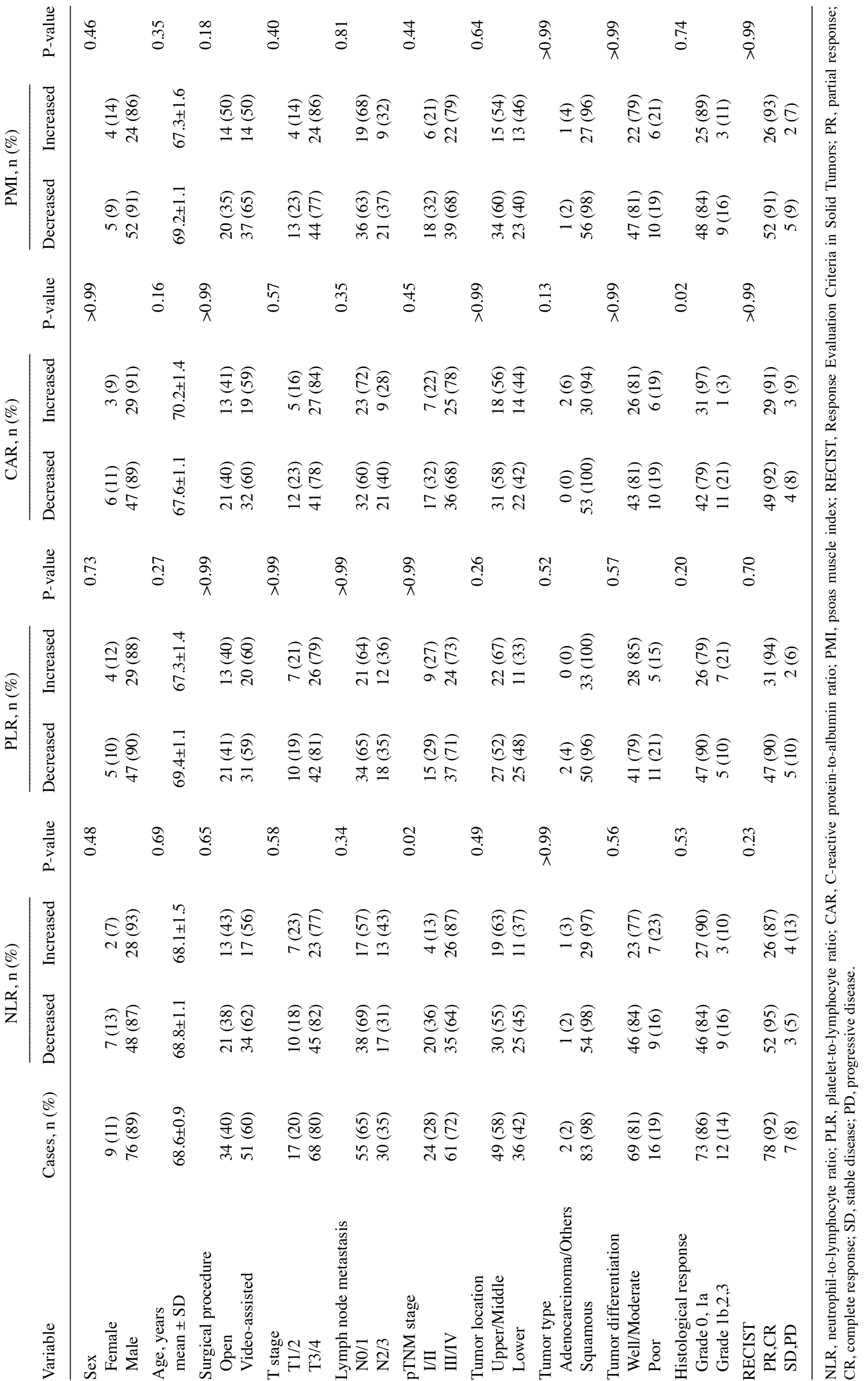




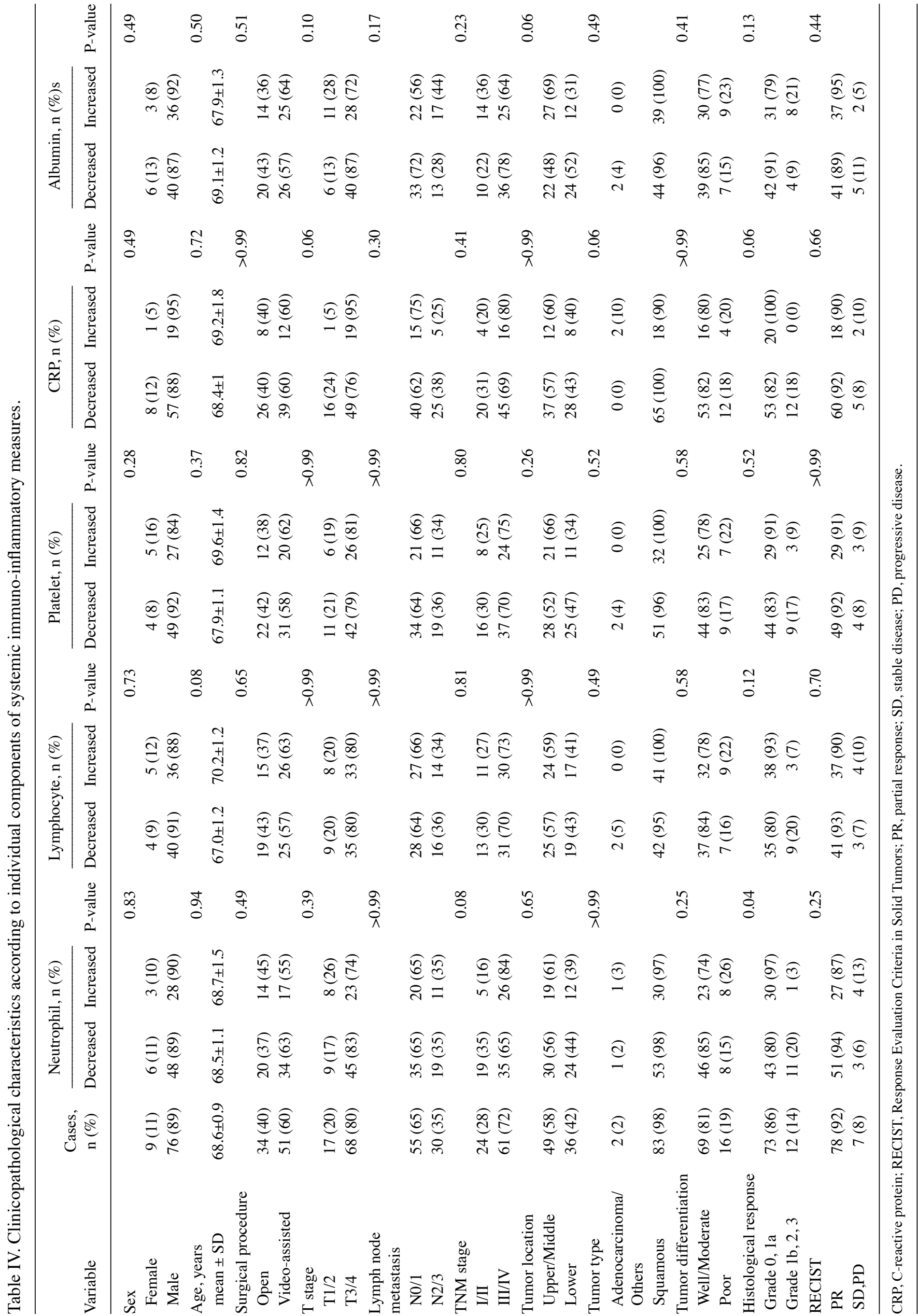


NLR

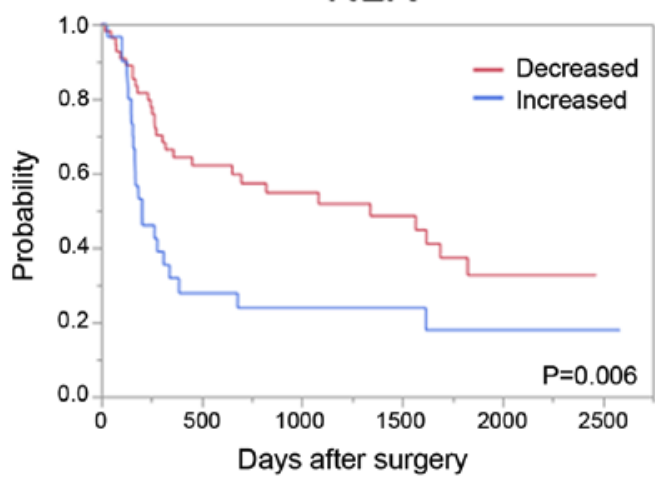

CAR

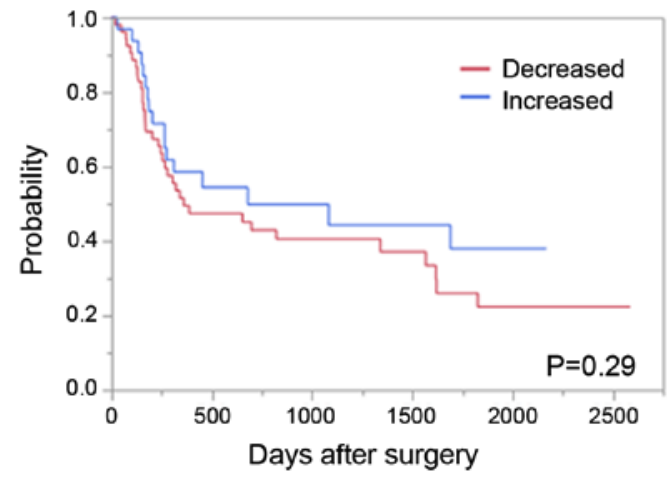

PLR

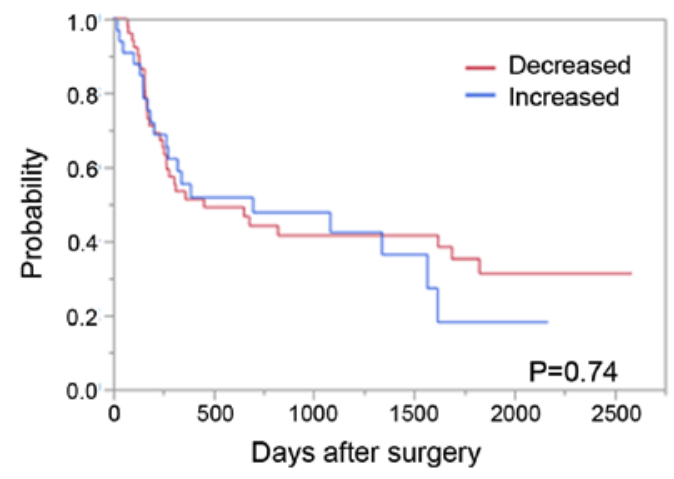

PMI

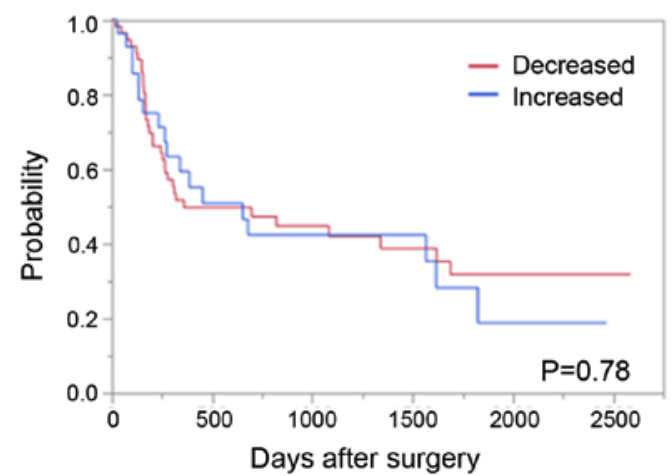

Figure 3. Overall survival rate following esophagectomy for esophageal cancer according to the alterations of systemic immuno-inflammatory measures during NAC. NLR, neutrophil-to-lymphocyte ratio; PLR, platelet-to-lymphocyte ratio; CAR, C-reactive protein-to-albumin ratio; PMI, psoas muscle index; NAC, neoadjuvant chemotherapy.
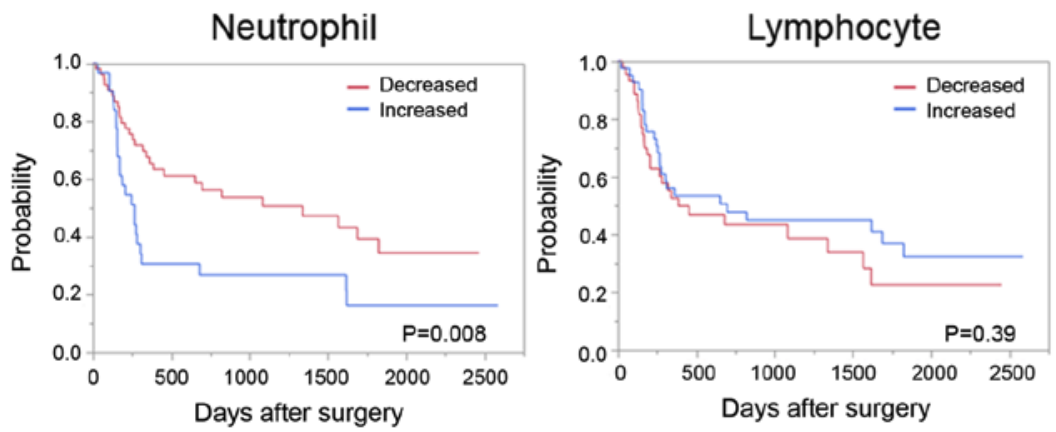

Platelet

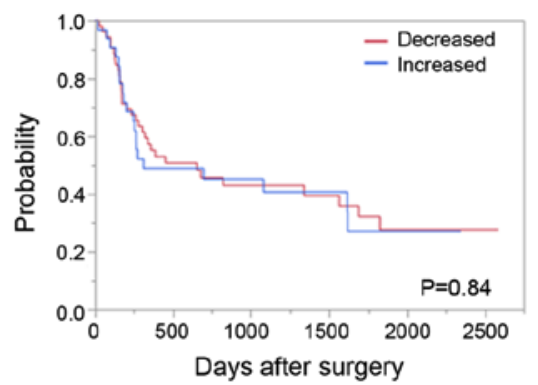

Albumin

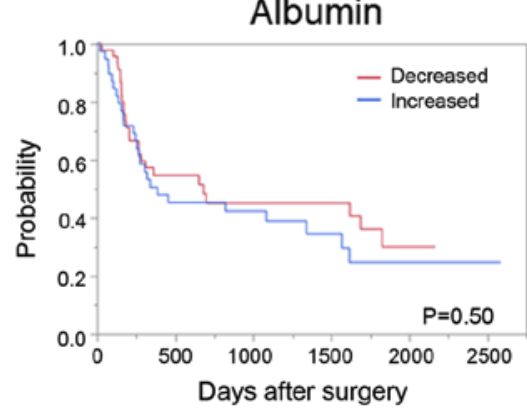

Figure 4. Overall survival rate following esophagectomy for esophageal cancer according to the alterations in neutrophils, platelets, lymphocytes, CRP levels and albumin levels during NAC. CRP, C-reactive protein; NAC, neoadjuvant chemotherapy.

measures (Table V) and their individual components (Table VI) due to their confounding. In the univariate analysis, the pathological stage and decreased NLR after NAC were significantly associated with favorable OS (Table V). The multivariate analysis demonstrated that only a decreased NLR after NAC was significantly associated with favorable OS. Both the 
Table V. Univariate and multivariate analyses for association between overall survival of patients with esophageal cancer and NLR, PLR CAR and PMI.

\begin{tabular}{|c|c|c|c|c|}
\hline Variable & Univariate analysis, HR (95\% CI) & P-value & Multivariate analysis, $\mathrm{HR}(95 \% \mathrm{CI})$ & P-value \\
\hline \multicolumn{5}{|l|}{ Sex } \\
\hline Female & Ref. & 0.420 & & \\
\hline Male & $1.483(0.603-4.920)$ & & & \\
\hline Age, years+1 & $2.285(0.473-12.45)$ & 0.320 & & \\
\hline \multicolumn{5}{|c|}{ Surgical procedure } \\
\hline Open & Ref. & 0.830 & & \\
\hline Video-assisted & $0.944(0.543-1.663)$ & & & \\
\hline \multicolumn{5}{|l|}{ p stage } \\
\hline Stage1-2 & Ref. & 0.030 & Ref. & 0.070 \\
\hline Stage 3-4 & $2.06(1.093-4.227)$ & & $1.811(0.948-3.752)$ & \\
\hline \multicolumn{5}{|l|}{ Tumor location } \\
\hline Lower/middle & Ref. & 0.210 & & \\
\hline Upper & $1.554(0.735-2.978)$ & & & \\
\hline \multicolumn{5}{|c|}{ Tumor differentiation } \\
\hline Well/moderate & Ref. & 0.080 & & \\
\hline Poor & $1.794(0.915-3.300)$ & & & \\
\hline \multicolumn{5}{|c|}{ Histopathological response } \\
\hline Grade 1a & Ref. & 0.650 & & \\
\hline$>$ Grade $1 \mathrm{~b}$ & $1.181(0.538-2.316)$ & & & \\
\hline \multicolumn{5}{|l|}{ NLR } \\
\hline Increased & Ref. & 0.009 & Ref. & 0.020 \\
\hline Decreased & $0.468(0.270-0.822)$ & & $0.5200(0.296-0.920)$ & \\
\hline \multicolumn{5}{|l|}{ PLR } \\
\hline Increased & Ref. & 0.749 & & \\
\hline Decreased & $0.9099(0.521-1.623)$ & & & \\
\hline \multicolumn{5}{|l|}{ CAR } \\
\hline Increased & Ref. & 0.301 & & \\
\hline Decreased & $1.357(0.771-2.482)$ & & & \\
\hline \multicolumn{5}{|l|}{ PMI } \\
\hline Increased & Ref. & 0.790 & & \\
\hline Decreased & $0.92(0.528-1.673)$ & & & \\
\hline
\end{tabular}

HR, hazard ratio; Ref., reference; pStage, pathological stage; NLR, neutrophil-to-lymphocyte ratio; PLR, platelet-to-lymphocyte ratio; CAR, C-reactive protein-to-albumin ratio; PMI, psoas muscle index.

univariate and multivariate analyses, including the individual components of the systemic immuno-inflammatory parameters demonstrated that the pathological stage and decreased neutrophil count after NAC were significantly associated with favorable OS (Table VI).

\section{Discussion}

The present study is the first report to evaluate the predictive value of the changes of various systemic immuno-inflammatory and nutritional parameters on the therapeutic effects of NAC and long-term outcomes in patients with esophageal cancer. In this study, we demonstrated that patients with high neutrophil counts before NAC had better histopathological responses to NAC. Additionally, we reported that decreases in the NLR and neutrophil counts after NAC were significant predictors of OS in patients with resectable stage II/III esophageal cancer.
Although many studies have shown that systemic immuno-inflammatory and nutritional parameters are useful predictors of long-term outcomes, there are conflicting results between these parameters and the response to $\operatorname{NAC}(5,17,18)$. Sato et al (19) reported that esophageal cancer patients with a lower NLR before NAC had a better response to NAC than those with a higher NLR. There have been similar reports for several malignancies (20,21). Conversely, Lorente et al (22) and Graziano et al (23) did not find any significance of NLR as a predictor of the response to NAC in breast cancer. In addition, our present study demonstrated that a pretherapeutic higher values of NLR and neutrophil count were related to a better response to NAC in esophageal cancer patients. In addition, we previously reported that the pretherapeutic higher values of NLR and CAR were associated with unfavorable outcome (11). It is well known that immuno-inflammatory parameters are easily affected by various factors, such as obstruction of the 
Table VI. Univariate and multivariate analyses of overall survival of patients with esophageal cancer and neutrophil, lymphocyte, platelet, CRP and Alb levels.

\begin{tabular}{|c|c|c|c|c|}
\hline Variable & Univariate analysis, HR (95\% CI) & P-value & Multivariate analysis, HR (95\% CI) & P-value \\
\hline \multicolumn{5}{|l|}{ Sex } \\
\hline Female & Ref. & 0.420 & & \\
\hline Male & $1.483(0.603-4.920)$ & & & \\
\hline Age (years+1) & $2.285(0.473-12.45)$ & 0.320 & & \\
\hline \multicolumn{5}{|c|}{ Surgical procedure } \\
\hline Open & Ref. & 0.830 & & \\
\hline Video-assisted & $0.944(0.543-1.663)$ & & & \\
\hline \multicolumn{5}{|l|}{$\mathrm{p}$ stage } \\
\hline Stage1-2 & Ref. & 0.030 & Ref. & 0.049 \\
\hline Stage3-4 & $2.06(1.093-4.227)$ & & $1.899(1.002-3.913)$ & \\
\hline \multicolumn{5}{|l|}{ Tumor location } \\
\hline Lower/middle & Ref. & 0.210 & & \\
\hline Upper & $1.554(0.735-2.978)$ & & & \\
\hline \multicolumn{5}{|c|}{ Tumor differentiation } \\
\hline Well/moderate & Ref. & 0.080 & & \\
\hline Poor & $1.794(0.915-3.300)$ & & & \\
\hline \multicolumn{5}{|c|}{ Histopathological response } \\
\hline Grade 1a & Ref. & 0.650 & & \\
\hline$>$ Grade $1 \mathrm{~b}$ & $1.181(0.538-2.316)$ & & & \\
\hline \multicolumn{5}{|l|}{ Neutrophil } \\
\hline Increased & Ref. & 0.010 & Ref. & 0.022 \\
\hline Decreased & $0.484(0.279-0.846)$ & & $0.519(0.298-0.910)$ & \\
\hline \multicolumn{5}{|l|}{ Lymphocyte } \\
\hline Increased & Ref. & 0.395 & & \\
\hline Decreased & $1.268(0.731-2.206)$ & & & \\
\hline \multicolumn{5}{|l|}{ Platelet } \\
\hline Increased & Ref. & 0.841 & & \\
\hline Decreased & $0.944(0.544-1.678)$ & & & \\
\hline \multicolumn{5}{|l|}{ CRP (mg/dl) } \\
\hline Increased & Ref. & 0.175 & & \\
\hline Decreased & $0.641(0.354-1.232)$ & & & \\
\hline \multicolumn{5}{|l|}{ Alb } \\
\hline Increased & Ref. & 0.504 & & \\
\hline Decreased & $0.830(0.479-1.436)$ & & & \\
\hline
\end{tabular}

HR, hazard ratio; Ref., reference; pStage, pathological stage; CRP, C-reactive protein; Alb, albumin.

tumor, anti-platelet drug use, smoking history, and co-morbidities (11). Thus, we believe that it is important to examine the changes in immuno-inflammatory parameters modulated by NAC because there is a limitation to predicting the response of NAC by these parameters just before NAC.

Konishi et al (24) reported that the severe neutrophil decline after NAC is associated with a high histological response in esophageal cancer, which is consistent with our present results. There are also several reports describing how hematological toxicity is correlated with a better response to NAC in breast and colorectal cancers $(25,26)$. The therapeutic effects of NAC for cancer cells depend on whether a sufficient amount of therapeutic agents can reach the cancer cells or whether the tumor is sensitive to these agents. The same is true of healthy cells, particularly hematopoietic cells. Thus, it is reasonable that hematological toxicity is correlated with the response to chemotherapy (27).

In the present study, we presented that a significant decline in the NLR after NAC was associated with favorable OS and a better NAC response in patients with esophageal cancer. Lee et al (28) also demonstrated that a significant reduction in the NLR after chemotherapy is associated with a better tumor response and a favorable outcome in advanced lung cancer patients. However, Konishi et al (24) did not find a correlation between the grade of neutropenia and survival outcome, although the severe neutrophil decline after NAC was correlated with a high histological response. For this reason, they speculated that severe neutropenia itself caused treatment-related death and was associated with worse long-term outcomes. 
PMI is a nutritional parameter and a marker of sarcopenia that was recently reported for its prognostic value in many malignancies $(29,30)$. Liu et al (31) reported that a declined PMI after NAC was associated with a poor prognosis in patients with esophageal cancer. In our previous study, we found that decline of PMI until 6 months after esophagectomy was independently associated with the incidence of pneumonia 6 months after resection for esophageal cancer (32). In this study, NAC decreased the PMI, but we could not find the relationship between changes in the PMI during NAC and survival outcomes.

The present study was conducted at a single institution using a retrospective design, a relatively small number of patients, and was not included the dose intensity and non-hematological adverse events of NAC, which are limitations of this study. Therefore, prospective studies with more patients are warranted to validate the results of this study.

In conclusion, among various systemic immunoinflammatory and nutritional parameters, a decreased NLR and decreased neutrophil counts after NAC had clinical significance as predictors of the response to NAC and of survival, respectively, in esophageal cancer. Predicting responses to NAC at the early phase of treatment, especially selecting non-responders, is significantly important to reduce unnecessary chemotherapy or convert the treatment to surgery before tumor progression. The systemic immune-inflammatory and nutritional measures may help predicting the efficacy of NAC to carefully investigate these changes during NAC.

\section{Acknowledgements}

Not applicable.

\section{Funding}

No funding was received.

\section{Availability of data and materials}

The datasets used and/or analyzed during the present study are available from the corresponding author on reasonable request.

\section{Authors' contributions}

YIs and HT conceived and designed the study. KK, ST, YIt, NI, SN, YY, HH, ES, YK, SH and HU contributed to data acquisition, data analysis and manuscript preparation. YIs, HT, $\mathrm{HH}, \mathrm{ES}, \mathrm{YK}$ and HU contributed to writing of the manuscript and provided supervision of the manuscript. All authors read and approved the final version of the manuscript.

\section{Ethics approval and consent to participate}

The Institutional Review Board of the National Defense Medical College Hospital approved this protocol. All patients provided written informed consent prior to their inclusion in the present study.

\section{Patient consent for publication}

Not applicable.

\section{Competing interests}

The authors declare that they have no competing interests.

\section{References}

1. Pisani P, Parkin DM, Bray F and Ferlay J: Estimates of the worldwide mortality from 25 cancers in 1990. Int J Cancer 83: 18-29, 1999.

2. Enzinger PC and Mayer RJ: Esophageal cancer. N Engl J Med 349: 2241-2252, 2003.

3. Bray F, Ferlay J, Laversanne M, Brewster DH, Gombe Mbalawa C, Kohler B, Piñeros M, Steliarova-Foucher E, Swaminathan R, Antoni S, et al: Cancer Incidence in five continents: Inclusion criteria, highlights from volume $\mathrm{X}$ and the global status of cancer registration. Int J Cancer 137: 2060-2071, 2015.

4. Ando N, Kato H, Igaki H, Shinoda M, Ozawa S, Shimizu H, Nakamura T, Yabusaki H, Aoyama N, Kurita A, et al: A randomized trial comparing postoperative adjuvant chemotherapy with cisplatin and 5-fluorouracil versus preoperative chemotherapy for localized advanced squamous cell carcinoma of the thoracic esophagus (JCOG9907). Ann Surg Oncol 19: 68-74, 2012.

5. Yodying $\mathrm{H}$, Matsuda A, Miyashita M, Matsumoto S, Sakurazawa N, Yamada M and Uchida E: Prognostic significance of neutrophil-to-lymphocyte ratio and platelet-to-lymphocyte ratio in oncologic outcomes of esophageal cancer: A systematic review and meta-analysis. Ann Surg Oncol 23: 646-654, 2016.

6. Wei XL, Wang FH, Zhang DS, Qiu MZ, Ren C, Jin Y, Zhou YX, Wang DS, He MM, Bai L, et al: A novel inflammation-based prognostic score in esophageal squamous cell carcinoma: The C-reactive protein/albumin ratio. BMC Cancer 15: 350, 2015.

7. Shimada H, Takiguchi N, Kainuma O, Soda H, Ikeda A, Cho A, Miyazaki A, Gunji H, Yamamoto H and Nagata M: High preoperative neutrophil-lymphocyte ratio predicts poor survival in patients with gastric cancer. Gastric Cancer 13: 170-176, 2010.

8. Sarraf KM, Belcher E, Raevsky E, Nicholson AG, Goldstraw P and Lim E: Neutrophil/lymphocyte ratio and its association with survival after complete resection in non-small cell lung cancer. J Thorac Cardiovasc Surg 137: 425-428, 2009.

9. Nagata T, Nakase Y, Nakamura K, Sougawa A, Mochiduki S, Kitai S and Inaba S: Prognostic impact of a nutritional index including muscle volume in stage 4 colorectal cancer. In Vivo 30: 885-891, 2016.

10. Kinoshita A, Onoda H, Imai N, Iwaku A, Oishi M, Tanaka K, Fushiya N, Koike K, Nishino $H$ and Matsushima M: The C-reactive protein/albumin ratio, a novel inflammation-based prognostic score, predicts outcomes in patients with hepatocellular carcinoma. Ann Surg Oncol 22: 803-810, 2015.

11. Ishibashi Y, Tsujimoto H, Hiraki S, Kumano I, Yaguchi Y, Horiguchi H, Nomura S, Ito N, Shinto E, Aosasa S, et al: Prognostic value of preoperative systemic immunoinflammatory measures in patients with esophageal cancer. Ann Surg Oncol 25: 3288-3299, 2018

12. Brierley JD, Gospodarowicz MK and Wittekind C (eds): TNM classification of malignant tumours. John Wiley \& Sons, 2016.

13. Tsujimoto $\mathrm{H}$, Hiraki $\mathrm{S}$, Takahata R, Nomura $\mathrm{S}$, Ito $\mathrm{N}$, Kanematsu K, Horiguchi H, Aosasa S, Yamamoto J and Hase K: Laparoscopic jejunostomy for obstructing upper gastrointestinal malignancies. Mol Clin Oncol 3: 1307-1310, 2015.

14. Hiraoka A, Aibiki T, Okudaira T, Toshimori A, Kawamura T, Nakahara H, Suga Y, Azemoto N, Miyata H, Miyamoto Y, et al: Muscle atrophy as pre-sarcopenia in Japanese patients with chronic liver disease: Computed tomography is useful for evaluation. J Gastroenterol 50: 1206-1213, 2015.

15. Tsujimoto H, Takahata R, Nomura S, Yaguchi Y, Kumano I, Matsumoto Y, Yoshida K, Horiguchi H, Hiraki S, Ono S, et al: Video-assisted thoracoscopic surgery for esophageal cancer attenuates postoperative systemic responses and pulmonary complications. Surgery 151: 667-673, 2012.

16. Tsujimoto H, Ono S, Sugasawa H, Ichikura T, Yamamoto J and Hase K: Gastric tube reconstruction by laparoscopy-assisted surgery attenuates postoperative systemic inflammatory response after esophagectomy for esophageal cancer. World J Surg 34: 2830-2836, 2010.

17. Xu XL, Yu HQ, Hu W, Song Q and Mao WM: A Novel inflammation-based prognostic score, the C-reactive protein/albumin ratio predicts the prognosis of patients with operable esophageal squamous cell carcinoma. PLoS One 10: e0138657, 2015. 
18. Duan $\mathrm{H}$, Zhang $\mathrm{X}$, Wang FX, Cai MY, Ma GW, Yang $\mathrm{H}$, Fu JH, Tan ZH, Meng YQ, Fu XY, et al: Prognostic role of neutrophil-lymphocyte ratio in operable esophageal squamous cell carcinoma. World J Gastroenterol 21: 5591-5597, 2015.

19. Sato H, Tsubosa Y and Kawano T: Correlation between the pretherapeutic neutrophil to lymphocyte ratio and the pathologic response to neoadjuvant chemotherapy in patients with advanced esophageal cancer. World J Surg 36: 617-622, 2012.

20. Feng JF, Huang Y and Chen QX: Preoperative platelet lymphocyte ratio (PLR) is superior to neutrophil lymphocyte ratio (NLR) as a predictive factor in patients with esophageal squamous cell carcinoma. World J Surg Oncol 12: 58, 2014.

21. Qian Y, Tao J, Li X, Chen H, Lu Q, Yang J, Pan H, Wang C, Zhou W and Liu X: Peripheral inflammation/immune indicators of chemosensitivity and prognosis in breast cancer patients treated with neoadjuvant chemotherapy. Onco Targets Ther 11: 1423-1432, 2018.

22. Lorente D, Mateo J, Templeton AJ, Zafeiriou Z, Bianchini D, Ferraldeschi R, Bahl A, Shen L, Su Z, Sartor O and de Bono JS: Baseline neutrophil-lymphocyte ratio (NLR) is associated with survival and response to treatment with second-line chemotherapy for advanced prostate cancer independent of baseline steroid use. Ann Oncol 26: 750-755, 2015.

23. Graziano V, Grassadonia A, Iezzi L, Vici P, Pizzuti L, Barba M, Quinzii A, Camplese A, Di Marino P, Peri M, et al Combination of peripheral neutrophil-to-lymphocyte ratio and platelet-to-lymphocyte ratio is predictive of pathological complete response after neoadjuvant chemotherapy in breast cancer patients. Breast 44: 33-38, 2019.

24. Konishi H, Fujiwara H, Shiozaki A, Hiramoto H, Kosuga T, Komatsu S, Ichikawa D, Okamoto K and Otsuji E: Effects of neutropenia and histological responses in esophageal squamous cell carcinoma with neo-adjuvant chemotherapy. Int J Clin Oncol 21: 95-101, 2016.

25. Eryilmaz MK, Mutlu H, Salim DK, Musri FY, Tural D and Coskun HS: The neutrophil to lymphocyte ratio has a high negative predictive value for pathologic complete response in locally advanced breast cancer patients receiving neoadjuvant chemotherapy. Asian Pac J Cancer Prev 15: 7737-7740, 2014.
26. Schuell B, Gruenberger T, Kornek GV, Dworan N, Depisch D, Lang F, Schneeweiss B and Scheithauer W: Side effects during chemotherapy predict tumour response in advanced colorectal cancer. Br J Cancer 93: 744-748, 2005.

27. Saarto T, Blomqvist C, Rissanen P, Auvinen A and Elomaa I: Haematological toxicity: A marker of adjuvant chemotherapy efficacy in stage II and III breast cancer. Br J Cancer 75: 301-305, 1997.

28. Lee Y, Kim SH, Han JY, Kim HT, Yun T and Lee JS: Early neutrophil-to-lymphocyte ratio reduction as a surrogate marker of prognosis in never smokers with advanced lung adenocarcinoma receiving gefitinib or standard chemotherapy as first-line therapy. J Cancer Res Clin Oncol 138: 2009-2016, 2012

29. Taguchi S, Akamatsu N, Nakagawa T, Gonoi W, Kanatani A, Miyazaki H, Fujimura T, Fukuhara H, Kume H and Homma Y: Sarcopenia evaluated using the skeletal muscle index is a significant prognostic factor for metastatic urothelial carcinoma. Clin Genitourin Cancer 14: 237-243, 2016.

30. Meza-Junco J, Montano-Loza AJ, Baracos VE, Prado CM, Bain VG, Beaumont C, Esfandiari N, Lieffers JR and Sawyer MB: Sarcopenia as a prognostic index of nutritional status in concurrent cirrhosis and hepatocellular carcinoma. J Clin Gastroenterol 47: 861-870, 2013.

31. Liu J, Motoyama S, Sato Y, Wakita A, Kawakita Y, Saito H and Minamiya Y: Decreased skeletal muscle mass after neoadjuvant therapy correlates with poor prognosis in patients with esophageal cancer. Anticancer Res 36: 6677-6685, 2016.

32. Nagata $K$, Tsujimoto $H$, Nagata $H$, Harada $M$, Ito $N$, Kanematsu K, Nomura S, Horiguchi H, Hiraki S, Hase K, et al: Impact of reduced skeletal muscle volume on clinical outcome after esophagectomy for esophageal cancer: A retrospective study. Medicine (Baltimore) 97: e11450, 2018.

This work is licensed under a Creative Commons Attribution-NonCommercial-NoDerivatives 4.0 International (CC BY-NC-ND 4.0) License. 